\title{
CULTURA ORGANIZACIONAL EM UMA EMPRESA FAMILIAR
}

\author{
ORGANIZATION CULTURE IN A FAMILY BUSINESS
}

\author{
Renan Cláudio dos Santos ${ }^{\mathrm{I}}$ \\ Fabiana Theodoro de Souza Bueno ${ }^{\text {II }}$
}

\begin{abstract}
RESUMO
Este trabalho teve como tema de pesquisa a cultura organizacional no contexto de uma empresa familiar e cujo objetivo geral foi averiguar a influência da cultura organizacional em uma empresa familiar. Este tema foi escolhido devido à oportunidade de apresentar o que acontece no ambiente de trabalho com relação à cultura local. A metodologia foi qualitativa, descritiva e um estudo de caso foi utilizado, em que os dados foram coletados por meio de uma entrevista com roteiro semiestruturado e analisados por meio da análise interpretativa, feito com o Gestor Operacional Corporativo (GOC), escolhido cuidadosamente entre outros, pois possui conhecimento amplo da equipe e dos processos organizacionais da organização, embora não faça parte da família do fundador. Os resultados apontaram que a empresa em estudo neste artigo, a qual será denominada de Empresa $X$, possui uma cultura forte e que valoriza o colaborador. Conclui-se que a cultura organizacional exerce influência em uma empresa familiar, pois solidifica as características e valores da organização e fortalece na criação da identidade, o que pode garantir vida longa mesmo que o fundador ou alguns membros se afastem da gestão.
\end{abstract}

Palavras-chave: Cultura Organizacional. Empresa. Familiar.

\begin{abstract}
This work was fulfilled through research on organizational culture in a family business context and aimed to find out the influence of the organizational culture on this type of business. This theme has been chosen due to the opportunity to present what happens in the workplace regarding local culture. The methodology used was qualitative, descriptive and a case study was used as well, where data was collected through a semi-structured script interview (carried out with the corporate operational manager who even though is not a member of the founder's family, knows a lot about all the team and the organizational processes) and then analyzed (interpretative analysis). The results showed that the company " $\mathrm{x}$ " has a strong culture and values its employees. It was concluded that the organizational culture influences the family business because it solidifies the company's characteristics and values strengthening identity creation which can ensure long life even if the founder or some of the members are away from management.
\end{abstract}

Keywords: Organization Culture. Business. Family.

\footnotetext{
I Tecnólogo em Gestão Empresarial pela Faculdade de Tecnologia (FATEC) de Sertãozinho - São Paulo Brasil. E-mail: renan6santos@outlook.com

II Professora Especialista da Faculdade de Tecnologia (FATEC) de Sertãozinho - São Paulo - Brasil. E-mail: fabianatheodorodesouza@bol.com.br
} 


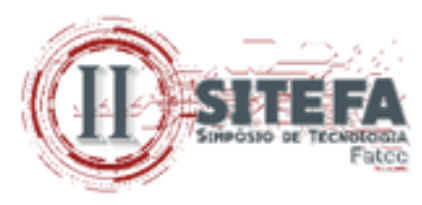

Data de submissão: 15/07/2019.

Data de aprovação do artigo: 01/10/2019.

DOI:

\section{INTRODUÇÃO}

Este trabalho tem como tema a cultura organizacional no contexto de uma empresa familiar, pelo fato de esse tipo de organização possuir características singulares, como a presença do fundador e seus familiares no mesmo ambiente. Assim sendo, é natural que uma empresa possua fatores que a identifiquem perante o público interno e externo, contudo, nas empresas familiares estas características são acentuadas devido a presença da família no local de trabalho.

Neste âmbito, Fidelis (2015) explica que, nas empresas familiares, existe uma crença de que quando a família e a empresa se unem, ocorre um enfraquecimento da eficiência da gestão dos negócios; ainda que essa ideia não seja assimilada por todos, pois muitas organizações tiveram um início marcado pela gestão familiar e hoje são grandes instituições.

Não existe uma fórmula que assegure o sucesso de uma empresa familiar, contudo, é preciso que os gestores consigam separar assuntos de família daqueles típicos de ambiente de negócios. A gestão deve ser estratégica, com espaço para críticas e sugestões, evitando o paternalismo e protecionismo que impera nesse cenário.

Associando esses aspectos à cultura organizacional, cabe trazer o conceito de Seldin, Rainho e Caulliraux (2019 - 2003, p.27) "cultura é o ato, efeito ou modo de cultivar e, ainda, o complexo dos padrões de comportamento das crenças, das instituições, das manifestações culturais, intelectuais etc., transmitidos coletivamente, e típicos de uma sociedade".

Isso significa que cada empresa, independente do porte que possua, tem a sua cultura, e a força que cada uma possui, é importante para a continuidade da empresa. Culturas fracas não sustentam longos períodos de crise, enquanto as fortes preparam o ambiente para lidar com mudanças econômicas e estruturais (SCHEIN, 2009).

Com base nas informações apresentadas, o problema de pesquisa é: Qual a influência da cultura organizacional em uma empresa familiar?

Corroborando, o estudo definiu que o objetivo geral seria averiguar a influência da cultura organizacional em uma empresa familiar.

A escolha do tema justifica-se pelo fato de o pesquisador atuar em uma empresa familiar e identificar, no ambiente de trabalho, uma oportunidade de abordar o tema, a fim de gerar conteúdo prático e que possa servir para consultas organizacionais. Acredita-se que, dessa forma, o trabalho consiga atingir seus objetivos e promover o debate acadêmico, porque o cenário de uma empresa familiar é rico em conhecimento e estratégias de negócios.

\section{CULTURA ORGANIZACIONAL}

Segundo Fleury e Sampaio (2002), a cultura organizacional pode ser definida como um conjunto de valores e pressupostos básicos, os quais são expressos por meio de elementos simbólicos que possuem a capacidade de ordenar, atribuir significações e construir a identidade organizacional. Podem também agir como elementos de comunicação e consenso, até mesmo ocultar e instrumentalizar as relações de dominação. 


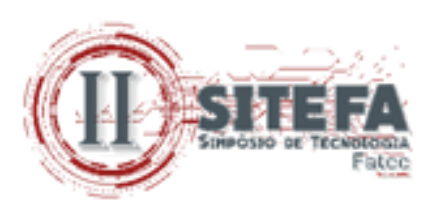

A cultura faz parte de um nível mais profundo de comportamento dos grupos, o que a diferencia do clima organizacional. Este, por sua vez, reflete a percepção de um grupo num determinado momento. Complementando, Chiavenato (2005) afirma que o clima organizacional é a qualidade percebida do ambiente organizacional, por meio das opiniões dos participantes da empresa, e exerce influência no comportamento dos colaboradores. Destacase, ainda, que o clima abrange as propriedades motivacionais do ambiente organizacional, revelando os aspectos internos da organização que podem levar à provocação de diferentes espécies de motivação nos seus participantes.

Assim sendo, Seldin Rainho e Caulliraux (2003) afirma que a cultura organizacional é considerada um elemento-chave para definir a posição que a empresa deverá adotar para que possa reter os melhores talentos, contribuindo para promover um ambiente propício para que o desenvolvimento da organização se concretize, mantendo uma relação harmoniosa entre as práticas e valores existentes. Dessa forma, entende-se que a difusão da cultura organizacional, por meio dos gestores, auxilia no alcance dos objetivos da organização.

Em relação às funções exercidas pela cultura organizacional, destacam-se as seguintes: 1 - fornecer uma identidade organizacional aos seus participantes, para que os valores e normas sejam compartilhados entre os colaboradores, no intuito de gerar união e objetivos em comum; 2 - facilita o compromisso coletivo, porque cria um forte sentimento de participação, colaboração e compromisso mútuo; 3 - promove a estabilidade organizacional quando encoraja a cooperação e o trabalho em equipe, partindo do senso de união; 4 - molda o comportamento dos colaboradores e concede sentido ao ambiente de trabalho (WAGNER; HOLLENBECK, 2010).

Segundo Zago (2000), a cultura é originada dos modelos mentais coletivos que foram consolidados por meio da experiência e convivência comum dos participantes de um grupo social, em um ambiente estabelecido, e que atribuem configuração para a sua interpretação e representação de identidade. Assim, pode-se dizer que a cultura organizacional se manifesta nas atitudes e comportamentos dos colaboradores, o que torna possível concluir que o comportamento social de um grupo ou comunidade é manifestação da sua cultura.

\subsection{Tipos e níveis da cultura organizacional}

A cultura organizacional pode ser interpretada como o modo costumeiro ou tradicional de pensar e fazer as coisas, e representa as normas informais e não escritas que servem para orientar o comportamento dos colaboradores no dia a dia, com o intuito de atingir os objetivos organizacionais. É transmitida por meio do compartilhar de informações entre os participantes da empresa, de forma que os novos membros devam aprender e aceitar para que também sejam aceitos no ambiente de trabalho (CHIAVENATO, 2007).

Segundo Schein (2009), os tipos de cultura organizacional são: - cultura do poder, voltada ao alcance de resultados, com poucas regras e regulamentos e o poder geralmente está centrado no dono; - cultura dos papéis, é orientada por regras, cargos e funções definidas, o que torna a empresa burocrática, o poder é definido pelo nível hierárquico; - cultura das tarefas, possui flexibilidade e espaço para mudanças, e o foco está nos projetos, e o poder pertence àquele que consegue resolver os problemas; cultura de pessoas, coloca o ser humano em primeiro lugar e atua para manter os colaboradores motivados, ouvindo as suas opiniões e estimulando-os constantemente. 


\section{(II) SITEFA}

A cultura se caracteriza ainda como forte, fraca, adaptativa ou conservadora: 1- cultura forte: os valores são compartilhados entre todos os colaboradores, o que demonstra que a cultura é estruturada e influencia comportamentos; 2 - cultura fraca: caracteriza-se por permitir mudanças frequentes nos valores ou hábitos, o que pode ser observado em empresas jovens, que estão em busca de espaço no mercado e construção de identidade; 3 - cultura adaptativa: típica de empresas disponíveis a mudanças e inovação, sempre em busca de atualizações e adaptação, mas preservando a estabilidade e identidade organizacional; 4 cultura conservadora: esse tipo de empresa dificilmente mudará os hábitos e os valores, porque possui costumes e tradições com raízes profundas e, mesmo que ocorram mudanças no cenário exterior, a cultura permanece preservada (SCHEIN, 2009).

Quanto aos níveis da cultura organizacional, estes são divididos em artefatos, valores compartilhados e pressupostos.

- Artefatos: abrange aquilo que é visível no ambiente da organização, como a estrutura física, a arquitetura do local, as roupas e os rituais de comportamento visíveis, como as festas de final de ano ou comemoração de aniversário da organização. No entanto, essa dimensão pode ser enganadora, porque os dados são interpretativos, logo, dependem da percepção, sentimentos e ações de quem os interpreta (MORAIS, 2008).

- Valores compartilhados: esse nível está em uma camada mais profunda do que os artefatos, ou seja, não é totalmente perceptível como os artefatos. Os valores podem ser aqueles já fixados pelos fundadores da empresa, as normas e a filosofia do local, bem como outros valores importantes para a realização do trabalho (FREITAS, 2002).

- Pressupostos: constitui-se no terceiro nível da cultura e, devido à sua profundidade, revela aquilo que os participantes acreditam, ou seja, é algo intrínseco nas atividades e dificilmente é possível alterá-lo, pois engloba os sentimentos e crenças inconscientes, este é o propósito da organização como um todo (SCHEIN, 2009).

Em relação ao exposto, Cercal (2001) informa que, de forma geral, os artefatos, valores e pressupostos podem variar de uma organização para outra, porque cada empresa possui a sua cultura, e esta pode ser percebida de maneiras diferentes pelos indivíduos, os quais possuem visões e construções sociais distintas que visualizam e compreendem os níveis mencionados de formas diferentes. Diante do exposto, a próxima etapa abordará sobre a cultura organizacional em empresas familiares.

\subsection{Cultura organizacional em empresas familiares}

A construção da cultura organizacional é percebida por Zago (2013) como um processo de aprendizagem, pelo fato de promover a alteração e incorporação de conceitos e princípios, o que resulta em um caráter de redefinição cognitiva dos participantes da empresa, que referem, tipicamente, à aprendizagem de nível criativo/inovativo.

Sendo assim, se a organização possui uma cultura em que os valores e princípios são coerentes, certamente receberá respeito de seus membros e, em caso de uma gestão advinda de um profissional externo, o mesmo deve se adaptar à cultura do local, respeitando os artefatos, valores e pressupostos (CASILLAS; VÁZQUEZ; DÍAZ, 2007).

No contexto de uma empresa familiar, cabe informar que a cultura organizacional é formada por valores, crenças e símbolos, que exercem influência no comportamento das pessoas, no desempenho econômico, bem como nos processos de mudança organizacional, porque esse conceito é válido para todas as empresas (BARBOSA, 2002). 


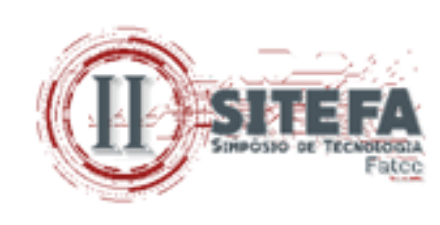

Sobre as características desse tipo de organização, Fleury, Ruas e Dutra (2008) destaca as seguintes: a gestão está ligada ao fundador, possui poucos níveis hierárquicos, laços afetivos, tratamento diferenciado entre os familiares e demais membros da empresa, proximidade com os gestores, visão compartilhada, presença de conflitos, confiança entre os membros da gestão, patrimonialismo.

Ressalta-se ainda a opinião de Leite (2013, p.56) sobre o tema abordado, "em sua origem, a cultura da organização, em geral, reflete a visão de seus fundadores, suas ideologias, costumes, gerando uma imagem de como a organização deveria ser". De acordo com Miorin (2014), assim como as demais organizações, a gestão familiar está sujeita a erros, em que os mais rotineiros são: a ausência de qualificação profissional, poder centralizado, misturar contas pessoais com as da empresa, zona de conforto, procrastinar o processo de sucessão, mais de um gestor na mesma área e evitar auxílio de consultorias e assessorias especializadas em administração.

Frente ao exposto, ressalta-se a importância de iniciar o preparo da sucessão empresarial enquanto o fundador ainda vive, a fim de conhecer os planos de sucessores, capacitar os mesmos e debater a possibilidade de aceitarem serem sócios um do outro. É importante ainda que os laços familiares não interfiram nas obrigações da empresa e viceversa. Dessa forma, é indicado que primeiro seja definido o modelo de sucessão que será implantado, para então dar início ao planejamento do processo sucessório (DUARTE; OLIVEIRA, 2010).

Para Schein (2009), a escolha de um novo gestor não deveria afetar a cultura organizacional, pois, caso ela seja forte, consegue subsistir mesmo quando alguns membros deixam a organização. Isso demonstra que a cultura é difícil de ser mudada, porque os participantes da empresa valorizam a estabilidade e reconhecem que ela fornece significado e previsibilidade.

Um resultado específico da cultura forte é percebido por meio dos índices de rotatividade da força de trabalho, os quais aparentam redução na presença da cultura citada, pois demonstra um elevado grau de concordância entre seus membros sob o ponto de vista da organização. A unanimidade de propósitos gera coesão, lealdade e comprometimento organizacional, e essas qualidades se encarregam de reduzir as possibilidades de os funcionários deixarem a organização (LEITE, 2013).

\section{PROCEDIMENTOS METODOLÓGICOS}

A pesquisa se classifica como sendo qualitativa e descritiva, sendo ainda um estudo de caso. Quanto à sua natureza, é uma pesquisa qualitativa, porque possibilita que o pesquisador tenha a facilidade de descrever a complexidade de um determinado problema, no intuito de apresentar contribuições no processo de mudança do tema abordado.

Do ponto de vista dos objetivos, o artigo se enquadra como descritivo, pois Cervo e Bervian (2000) explicam que esse método se caracteriza por observar, registrar, analisar e correlacionar fatos, mas evita manipulá-los. Dessa forma, a pesquisa descritiva busca identificar aspectos sobre os fenômenos observados, a fim de determinar a sua relação com outros.

No tocante aos procedimentos técnicos, trata-se de um estudo de caso, pois como informa Martins (2008, p. 11), permite "um mergulho profundo e exaustivo em um objeto 


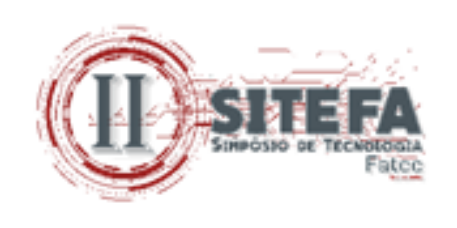

delimitado [...] possibilita a penetração em uma realidade social, não conseguida plenamente por um levantamento amostral e avaliação exclusivamente quantitativa".

A delimitação do estudo se encarrega de estabelecer limites para a investigação, e, neste aspecto, a pesquisa foi realizada em uma empresa familiar, de médio porte, contando com 362 colaboradores, localizada na cidade de Sertãozinho, SP.

Segundo dados do IBGE e do SEBRAE, 90\% das empresas no Brasil são familiares, representando cerca de $65 \%$ do PIB e empregando $75 \%$ da força de trabalho do país. (REVISTA EXAME, 2019).

A escolha da empresa se deu por conveniência, pois o pesquisador atua no local e tem fácil acesso aos gestores. A pesquisa foi realizada no mês de maio de 2019, na sede da empresa. Quanto à técnica de coleta de dados, definiu-se que seria a entrevista, elaborada com roteiro semiestruturado. Para Cervo e Bervian (2000), a entrevista não deve ser considerada uma simples conversa, pois tem objetivos definidos para ocorrer, tais como: recolher, por meio do interrogatório do entrevistado, conteúdo para a pesquisa.

Os dados provenientes da entrevista foram analisados por meio da análise interpretativa, método que procura dar um significado mais amplo às respostas, associando-as a outros conhecimentos. Esse recurso se caracteriza pela exposição do verdadeiro significado do material apresentado, comparando com os objetivos propostos e o tema abordado, fazendo ilações mais amplas dos dados discutidos (MARCONI; LAKATOS, 2010).

\section{RESULTADOS E DISCUSSÃO}

Esta etapa do artigo traz uma análise dos dados coletados por meio da entrevista, a qual foi realizada na Empresa $X$, uma organização de transporte de cargas para o setor sucroenergético, que possui a sua matriz localizada na cidade de Sertãozinho-SP. Essa instituição expande-se em território brasileiro com onze filiais localizadas estrategicamente para atender à demanda. Fundada em 10 de dezembro de 2001, a empresa vem de constante crescimento desde seu início e atualmente tornou-se referência no mercado regional.

Dando início à análise dos dados coletados pela entrevista, a primeira pergunta indagou-se se a Empresa X é um lugar de cultura forte e, neste sentido, a resposta foi sim. Segundo o gestor, trata-se de uma empresa de capital fechado, familiar, constituída pelo bem de todos e valor às pessoas que sempre lutaram por ela. É reconhecida ainda por ser uma organização que zela pelo seu patriarca, e os valores transmitidos por ele aos filhos, os quais presidem a empresa hoje.

Comparando com a teoria, entende-se que a cultura do local pode sobreviver mesmo que alguns membros se afastem da empresa. Sendo a cultura algo difícil de ser modificado, os colaboradores tendem a valorizar a estabilidade que ela oferece, em virtude do significado e previsibilidade gerados (SCHEIN, 2009).

$\mathrm{Na}$ sequência, foi indagado se a empresa é percebida como sendo flexível, aberta à criatividade, inovações e mudanças. De acordo com o gestor entrevistado, o CEO da empresa apoia, investe e acredita em todos os intraempreendedores; compartilhando com eles suas experiências e trabalhando juntos em todas as inovações e/ ou mudanças. A empresa está sempre aberta às mais diversas possibilidades, buscando aproveitar o capital intelectual dos colaboradores visando a melhorar a convivência e seus processos e procurando melhorar e se adaptar ao mercado e às exigências. 


\section{(II) SITEFA}

Isso condiz com a opinião de Schein (2009), o qual explica que uma empresa com cultura marcada pela flexibilidade está sempre em busca de adaptação, sem que para isso precise descartar a estabilidade e a identidade da empresa.

$\mathrm{Na}$ pergunta seguinte, o entrevistado foi questionado se os gestores conseguem transmitir a cultura da empresa para os colaboradores. Novamente a resposta foi afirmativa, porque ainda que cada gestor sempre pense em inovações e busque realizar algumas mudanças, a raiz é preservada, e os princípios seguem sendo transmitidos aos novos colaboradores. No momento da integração do colaborador na empresa, já é ensinado como a mesma surgiu e como se manteve. Portanto, os gestores sempre conseguem transmitir a cultura da empresa, pois estão aptos e capacitados a realizar processos, resolver problemas e delegar tarefas; além disso, o gestor é responsável pela transmissão e entendimento dos valores para a sua equipe.

$\mathrm{Na}$ teoria, a cultura é considerada um elemento-chave para determinar a direção que a organização deve seguir, logo, se a cultura deixar de ser transmitida, a empresa perderá a capacidade de orientação e alcance de objetivos (SELDIN; RAINHO; CAULLIRAUX, 2003).

Em seguida, o entrevistado informou se a empresa possui símbolos que a caracterizem. Segundo o gestor, a disposição física da empresa está interligada para melhor fluência das informações que pode ser comparada com o descrito abaixo, parte essencial no desenvolvimento do espírito de equipe e comunicação.

Frente ao exposto, Morais (2008) afirma que os símbolos materiais são inspirados na estrutura física da organização, usando, para isso, a arquitetura do local e a mobília existente; logo, esses símbolos possuem a capacidade de gerar impacto nos indivíduos da organização.

Na sequência, a entrevista abordou questões sobre os rituais ou cerimônias, indagando se a empresa realiza algum. Conforme o gestor, a Empresa $\mathrm{X}$ realiza, no final de cada ano, uma cerimônia para comemorar o recente desempenho e dedicação dos colaboradores. Esse evento fica marcado por meio de uma série de premiações (monetárias e materiais) concedidas aos que mais se destacaram ao longo do ano.

Isso condiz com a teoria de Morais (2008) que explica que os rituais são descritos como eventos repetitivos que manifestam os mais importantes valores da organização, como a festa de final de ano. Sendo assim, os rituais podem impactar diretamente no clima organizacional, e como este está contido na cultura, pode sinalizar um ambiente onde predomina uma cultura de pessoas. As premiações são usadas como incentivo para aqueles que mais se dedicaram ao longo do ano, motivando o colaborador procurar sempre melhorar seu desempenho (SCHEIN, 2009).

A entrevista abordou sobre os valores da empresa, a fim de saber se eles são os mesmos desde a fundação da empresa ou se foram se adequando com o passar do tempo. Nesse âmbito, o gestor apontou que a Empresa $X$ se alicerça em: empreendedorismo, perseverança e coragem, inteligência, criatividade e aprendizado, ética, confiança e comprometimento. Esses valores são praticados entre todos os participantes, comunidade e mercado onde a organização atua e são os mesmos desde a fundação da empresa.

Os valores da empresa são uma raiz forte mantida desde a sua fundação e isso corresponde à opinião de Cercal (2001), porque o conjunto de artefatos, normas, valores e premissas se diferenciam de uma cultura para outra. Contudo, constituem-se como essenciais para a manutenção da cultura.

Sobre as principais normas da organização, o gestor informou que a Empresa X possui o "Regulamento de Pessoal" e "Regulamento de Comunicação". 


\section{(II) SITEFA}

Regulamento de Pessoal, como mencionado anteriormente, é um conjunto geral de direitos que visam a registrar de forma clara, as obrigações, deveres, valores e penalidades aplicáveis a cada indivíduo. Considera-se também como exemplo, uma série de quadros posicionados ao redor da empresa contendo os valores e frases motivacionais.

Regulamento de Comunicação, segundo o gestor, nada mais é do que a forma como os indivíduos dentro da organização devem se comunicar, uma maneira padronizada de realizar tarefas e transmitir informações claras para que qualquer colaborador entenda e seja capaz de agir em diversas situações, o que condiz com a afirmação de Cahen (2005) que salienta a comunicação empresarial como algo sério que deve ser usada como uma eficiente estratégia, que ainda é privilégio de poucos empresários, que aceitam a atividade como investimento e não como despesa.

Para finalizar, a entrevista abordou sobre os colaboradores, para saber se o empenho destes é percebido no alcance das metas organizacionais, e, ainda, se o esforço dos participantes pode ser tido como fruto da cultura existente na empresa.

Conforme o gestor, o investimento em pessoas é a maior fonte de lucro da Empresa X, e todos os colaboradores trabalham em congruência. Esse tem sido um dos maiores legados da organização, porque os colaboradores absorvem o espírito de trabalho em equipe em busca de promoções de seus cargos por meritocracia. Isso resulta em um melhor entendimento e trabalho de equipe em todos os setores, pois os funcionários sentem-se motivados e se empenham para melhorar os seus resultados.

Diante dessa informação, cabe trazer a visão de Leite (2013), pois uma evidência típica da cultura forte é um índice reduzido de rotatividade da força de trabalho. Assim, uma cultura forte revela a existência de um elevado grau de concordância entre seus membros, o que gera coesão, lealdade e comprometimento organizacional. E isso resulta na redução da rotatividade.

\section{CONCLUSÃO}

Este trabalho abordou a cultura organizacional no contexto de uma empresa familiar e, para tal, contou com uma entrevista realizada com o gestor operacional da organização, o qual possui um amplo conhecimento a respeito de diversos aspectos da organização, desde o pessoal da empresa e suas particularidades até os processos operacionais; portanto, foi possível coletar dados precisos, apresentados neste artigo, que introduzem e justificam a cultura organizacional existente na empresa e a influência familiar já estabelecida.

Com base nos resultados obtidos e analisados, destaca-se que a cultura organizacional presente em uma empresa familiar, em especial, a Empresa X, é uma cultura muito forte, e de presença constante. De acordo com o gestor, espera-se que os colaboradores conheçam de forma clara, qual é a raiz da empresa, ou seja, qual a cultura e os valores praticados no ambiente de trabalho. Tal conhecimento é essencial para o funcionamento da organização e o fluxo existente dentro de todos os setores.

A cultura organizacional existente apresentada na empresa orienta os profissionais a se adaptarem a um ambiente comum. Quando todos os colaboradores trabalham juntos e compreendem a importância de cada um dentro da organização, é notório que a empresa se beneficia muito desse aspecto para o seu crescimento, que, como foi apresentado pelo gerente operacional entrevistado, vem ocorrendo de forma constante nos últimos anos. 


\section{(1010)}

No final da pesquisa apresentada, é possível enxergar a influência da liderança familiar como algo positivo, uma forma de orientar e motivar os colaboradores, que, além disso, gera uma cultura organizacional forte e constante, também sendo essencial para a evolução da empresa, que já é referência regional em diversos estados, atendendo, em grande volume, a uma larga escala de clientes importantes e grande influência.

Sendo assim, todo colaborador ingressa no quadro de funcionários apenas com o perfil encaixando-se na cultura existente, isso é avaliado e estudado desde o início e, após o começo de suas atividades, é adaptado e orientado rapidamente por toda a equipe operacional.

Pode-se concluir, ainda que, devido a todos esses aspectos positivos mencionados, empresas que contam com uma cultura organizacional forte e familiar possuem maiores chances de sobreviver e adaptar-se ao mercado.

\section{REFERÊNCIAS}

BARBOSA, Lívia. Cultura e empresas. Rio de Janeiro: Jorge Zahar, 2002.

CAHEN, Roger. Comunicação Empresarial: tudo que seus gurus não lhe contaram sobre: 10. ed. Rio de Janeiro: Best Seller, 2005.

CASILlAS, J. C.; VÁZQUEZ, A.; DÍAZ, C. Gestão da empresa familiar: conceitos, casos e soluções. São Paulo: Thomson, 2007.

CERCAL, Marco Aurélio. Instrumento de avaliação da cultura organizacional. 2001. 129 p. Dissertação (Mestrado) - Universidade Federal de Santa Catarina, Florianópolis, 2001. Disponível em:

https://repositorio.ufsc.br/bitstream/handle/123456789/79661/185892.pdf?sequence=1\&isAll owed=y. Acesso em: 15 maio 2019.

CERVO, Amado L; BERVIAN, Pedro A. Metodologia Científica. 5. ed. São Paulo: Pearson Prentice Hall, 2000.

CHIA VENATO, Idalberto. Comportamento Organizacional: a dinâmica do sucesso das organizações. 2. ed. Rio de Janeiro: Elsevier, 2005.

Administração: teoria, processo e prática. 4. ed. Rio de Janeiro: Elsevier, 2007.

DUARTE, F. D.; OLIVEIRA, L. R. de. Análise de maturidade de processos sucessórios em empresas familiares. Rege, v. 17, n. 2, p. 135 - 150, 2010.

REVISTA EXAME. Empresas familiares assumem liderança de mercado. Disponível em: https://exame.abril.com.br/negocios/dino/empresas-familiares-assumem-lideranca-demercado/. Acesso em: 14 set. 2019.

FIDELIS, Isadora Carvalho. Conflitos em empresas familiares: um estudo de caso na empresa Gabrisa Aramados - Cafelândia SP. 2015. 57 fl. Monografia (Trabalho de Conclusão 
de Curso) - Centro Universitário Católico Salesiano Auxilium - UNISALESIANO, Lins, 2015. Disponível em:

http://www.unisalesiano.edu.br/biblioteca/monografias/58538.pdf. Acesso em: 21 maio 2019.

FLEURY, Maria Tereza Lemes; SAMPAIO, Jáder dos Reis. Uma discussão sobre cultura organizacional. In: FLEURY, Maria Tereza Lemes; SHINYASHIKI, Gilberto; FRANÇA, Ana Cristina Limongi; CASADO, Tania; FISCHER, Rosa Maria; JÚNIOR, Moacir de Miranda Oliveira; EBOLI, Marisa; ALBUQUERQUE, Lindolfo Galvão de; HIPÍLITO, José Antônio Monteiro; DUTRA, Joel de Souza; SAMPAIO, Jáder dos Reis; REIS, Germano Glufke; AURELIANO, Eliete Bernal; NOGUEIRA, Arnaldo José França Mazzei; FISCHER, André Luiz. As pessoas na organização. 16. ed. São Paulo: Gente, 2002.

FLEURY, Maria Tereza Lemes; RUAS, Roberto; DUTRA, Joel Souza. Competências Conceitos, Métodos e Experiências. São Paulo: Atlas, 2008.

FREITAS, M. E. Cultura organizacional: identidade, sedução e carisma? 3. ed. Rio de Janeiro: FGV, 2002.

LEITE, Paulo Marcel de Souza. A cultura organizacional na seleção de funcionários. Revista Ampla de Gestão Empresarial, v. 4, n. 2, p. 49 - 63, mai. 2013.

MARCONI, Marina de Andrade; LAKATOS, Eva Maria. Fundamentos de Metodologia Científica. 7. ed. São Paulo: Atlas, 2010.

MARTINS, G. A. Estudo de caso: uma reflexão sobre a aplicabilidade em pesquisas no Brasil. Revista de Contabilidade e Organizações, v. 2, n. 2, p. 9-18, jan./abr., 2008.

MIORIN, S. H. Choque de gestão na empresa familiar. Pet Center, Campinas, n.171, p. 9699, dez/2014.

MORAIS, Matheus Nunes. Cultura e subcultura: um estudo do setor de tecelagem da BETA S.A./JP. 2008. 51 fl. Monografia (Trabalho de Conclusão de Curso) - Centro Universitário de João Pessoa - UNIPÊ, João Pessoa, 2008.

SCHEIN, Edgar H. Cultura organizacional e liderança. São Paulo: Atlas, 2009.

SELDIN, Renata; RAINHO, Maria Alice Ferruccio; CAULLIRAUX, Heitor Mansur. O Papel da Cultura Organizacional na Implantação de Sistemas Integrados de Gestão: uma abordagem sobre resistência a mudanças. In: XXIII ENCONTRO NAC. DE ENG. DE PRODUÇÃO - Ouro Preto, MG, Brasil, 21 a 24 de out de 2003. Disponível em: http://www.abepro.org.br/biblioteca/enegep2003_tr0404_0850.pdf. Acesso em: 10 maio 2019.

WAGNER III, John A.; HOLLENBECK, John R. II. Comportamento Organizacional: criando vantagem competitiva. 3. ed. São Paulo: Saraiva, 2010. 
ZAGO, Célia Cristina. Modelo de arquitetura da cultura organizacional - MARCO: as dimensões da cultura Organizacional suportadas pela sua inter-relação com as variáveis do comportamento humano. 2000. 162 fl. Tese (Doutorado) - Universidade Federal de Santa Catarina - UFSC, Santa Catarina, 2000.

Cultura organizacional: formação, conceito e constituição. Revista Eletrônica Sistemas \& Gestão Volume 8, Número 2, 2013, pp. 106-117. Disponível em: http://www.revistasg.uff.br/index.php/sg/article/download/v8n2a1/v8n2a1. Acesso em: 21 maio 2019. 